\title{
Tyrosine-Protein Kinase Yes
}

National Cancer Institute

\section{Source}

National Cancer Institute. Tyrosine-Protein Kinase Yes. NCI Thesaurus. Code C18556.

Tyrosine-protein kinase Yes (543 aa, $\sim 61 \mathrm{kDa}$ ) is encoded by the human YES1 gene. This protein plays a role in both receptor tyrosine kinase-mediated signaling and tyrosine phosphorylation. 